\title{
Dissipation as a mechanism of energy gain
}

\author{
R. Egydio de Carvalho, ${ }^{1, *}$ C. Vieira Abud, ${ }^{1}$ and F. Caetano Souza ${ }^{2}$ \\ ${ }^{1}$ Departamento de Estatística, Matemática Aplicada e Computação-DEMAC, Instituto de Geociências e Ciências Exatas-IGCE, \\ Universidade Estadual Paulista-UNESP, Av. 24A, No. 1515, Bela Vista, 13506-900 Rio Claro, São Paulo, Brazil \\ ${ }^{2}$ Instituto de Física de São Carlos, Universidade de São Paulo, 13560-970 São Carlos, São Paulo, Brazil
}

(Received 29 August 2007; revised manuscript received 17 January 2008; published 7 March 2008)

\begin{abstract}
Some properties of the annular billiard under the presence of weak dissipation are studied. We show, in a dissipative system, that the average energy of a particle acquires higher values than its average energy of the conservative case. The creation of attractors, associated with a chaotic dynamics in the conservative regime, both in appropriated regions of the phase space, constitute a generic mechanism to increase the average energy of dynamical systems.
\end{abstract}

DOI: 10.1103/PhysRevE.77.036204

PACS number(s): 05.45.Pq, 05.90. $+\mathrm{m}$

\section{INTRODUCTION}

Many works on dissipative systems report results concerning the minimization or the elimination of the dissipation effects in different fields of science, such as molecular physics [1], optimal network [2,3], Raman lasers [4], turbulent fluid [5], viscous liquid [6], and Fermi quantum gas [7]. In an opposite approach, other works take advantage of the presence of dissipative effects and there are results reported in fields of science such as mesoscopic device [8], optics [9], relativity [10], nanoscience $[11,12]$, and nonlinear encryption [13].

In this paper we will follow the later approach by considering a nonlinear dynamical system where dissipative effects are present and, for a particular set of parameters, the system experiences a gain in its average energy. The common sense establishes that when dissipative effects are present in a system, it loses energy, what is correct in principle, but when chaos is also present in the dynamics, the combination with dissipation and attractors can produce this scenario. Our essential idea is to generate attractors, through bifurcations of elliptic fixed points in stable focus [14], for velocities which are higher than the typical average velocity of the system in the nondissipative regime. In the next section we present the model we are using and in Sec. III we show our results and the conclusions.

\section{MODEL}

We will revisit the time-dependent annular billiard $[15,16]$, but now under the presence of inelastic collisions with both boundaries. This work is a natural extension of the studies developed on Fermi acceleration where the pulsating annular billiard was used as model $[17,18]$. We will seek to understand and describe the behavior of the average velocity of a particle as a function of the collisions with the boundaries. The model consists of a circle circumscribed in another circle in whose annular region a classical particle moves freely. The boundaries defined by the circumferences play the role of infinite potential barriers. The geometry of the

\footnotetext{
*Corresponding author. regydio@rc.unesp.br
}

model may assume two configurations: (i) concentric circles or (ii) eccentric circles where the distance between both centers is called by eccentricity. For the nondissipative static case $[15,16]$, where both boundaries are static, the chaotic sea grows with the increasing of the eccentricity. For the limit case where the circles are concentric, the system is globally integrable because both the energy and the angular momentum are preserved. On the other hand, for the timedependent elastic case, the oscillations of the boundaries break the invariant tori and even for the concentric configuration it is possible to have a chaotic sea. In previous works $[17,18]$ we considered the boundaries of the annular billiard to pulsate periodically in time and now, in this work, we will take advantage of that approach to introduce inelasticity in the boundaries.

In the static elastic case, a particle moves in straight lines between two collisions with the circles and, at each collision, its energy and both the tangential and radial components of the linear momentum are preserved. The motion is governed by two nonlinear maps, one of them describes the dynamics when the particle does not hit the inner circle, between two consecutive collisions with the outer circle, and the other one foresees the collision with the inner circle. For both situations, the angle of incidence that the particle makes with the normal vector of any circle is the same as the angle that the particle exits from the collision, characterizing specular reflections. The corresponding phase space is essentially determined by two angles defined at the instant of each collision with the external circle. One of them is measured with respect to the normal vector of the outer circle and the other one is associated with the arc length counted from the abcissa axis. The reader may find out the details of the corresponding calculations in $[15,16]$.

For the elastic time-dependent case, the radii of the circles are functions of time given by $r(t)=r_{0}+\varepsilon_{r} \cos \left(\omega t+\phi_{n}\right)$ and $R(t)=1+\varepsilon_{R} \cos \left(t+\varphi_{n}\right)$, where $r_{0}$ and 1 correspond to the static radii of the inner and outer circles, respectively. The quantities $\phi_{n}$ and $\varphi_{n}$ are the oscillating phases of each boundary in the $n$th iteration of the maps, $\varepsilon_{r}$ and $\varepsilon_{R}$, are the boundaries amplitudes of oscillation, and $\omega$ is the ratio between the two frequencies. The regions $\left[r_{0}-\varepsilon_{r}, r_{0}+\varepsilon_{r}\right]$ and $\left[1-\varepsilon_{R}, 1+\varepsilon_{R}\right]$ define the collision zones for both the inner and outer circles. When the particle attains a collision zone it 
may suffer a collision. Depending on the velocity of the particle and on the phase of the moving boundary, at the entrance of a collision zone, it may suffer many others successive collisions before leaving it. It is possible to look at the problem of successive collisions through two versions, one called complete which consider all possibilities of collisions in the numeric calculations and another one, called simplified, which keeps the boundaries static but the particle changes its momentum as if the boundaries were oscillating. The computational calculations are faster in the simplified version and it is expected that the results are essentially similar as the ones from the complete version, thus we will consider the simplified version of the time-dependent dissipative annular billiard model in this paper.

Due to the symmetry of the billiard, the momentum transfer is similar to the central force problem, in such way that only the radial component of the momentum is changed at each collision. We will consider an index $n$ in order to identify the $n$th collision of the particle with the outer circle so that the corresponding values of the particle's velocity are given by

$$
\begin{gathered}
V_{(n+1) \eta}=-\left|-e V_{n \eta}+(1+e) U\right|, \\
V_{(n+1) \tau}=V_{n \tau},
\end{gathered}
$$

where $\eta$ and $\tau$ denote the radial and tangential components, respectively, $U$ is the velocity of the boundary which may be $\dot{R}(t)$ or $\dot{r}(t)$ depending on which boundary the collision happened, and the parameter $e \in[0,1]$ is the restitution coefficient. For $e=1$ there is no dissipation, see Fig. 1(a), but for $e \neq 1$ the areas on the phase space are not anymore conserved and attracting fixed points emerge, see Figs. 2(a) and 2(b).

\section{RESULTS AND CONCLUSIONS}

The inelastic time-dependent annular billiard has an expressive quantity of control parameters and after several simulations we have chosen the following parameter set: $r_{0}$ $=0.4, \omega=0.5, \varepsilon_{R}=\varepsilon_{r}=0.01$, and $\phi_{n}=\varphi_{n}$. We emphasize that this choice is representative for the annular billiard and that we will consider only the concentric case because in this nondissipative case it is well known that the particle has limited velocities due to the existence of spanning curves in the phase space $[17,18]$, which is convenient for the purpose of this work. This characteristic allows us to perform a more precise comparison among the scenarios determined by the values of $e$. When the boundaries oscillate, there is another phase plane defined by the pair of coordinates $\left(\varphi,-V_{\eta}\right)$ which is more appropriated to observe the behavior of the particle velocities. Figure 1(a) shows a plot of 20 initial conditions on the Poincare section $\left(\varphi,-V_{\eta}\right)$ for the case of null dissipation, $e=1$, and $\varphi \in[0,4 \pi]$, while Fig. 1(b) shows the corresponding plot for the average velocity as a function of the collision number $n$. In our simulations we have evolved the maps up to $10^{6}$ iterations and we observed that the average velocity saturates around $\langle V\rangle \approx 0.1$. On the other hand, in Fig. 2(a) we consider a great restitution coefficient $e$ $=0.9999$, characterizing a weakly dissipate system, which in
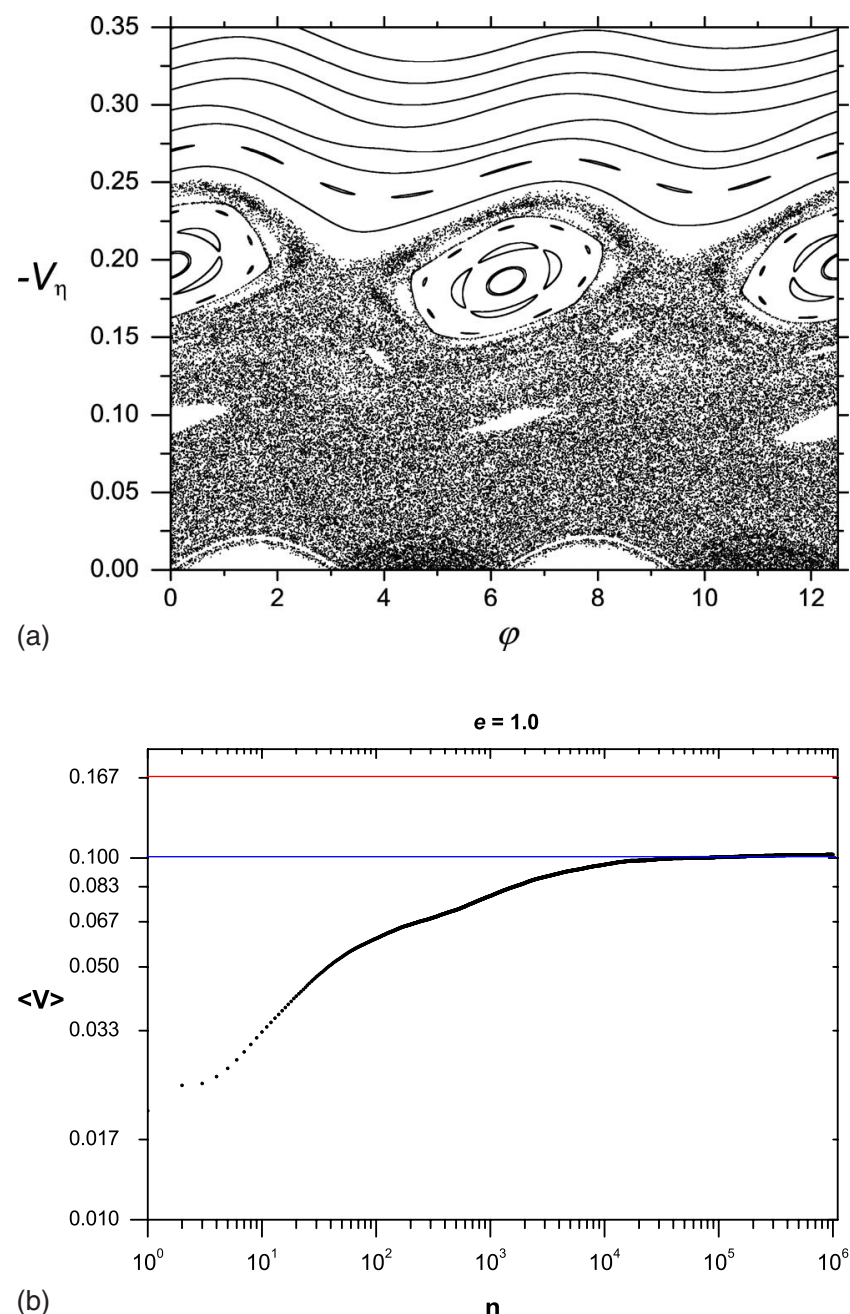

FIG. 1. (Color online) (a) Phase space for the concentric pulsating annular billiard representing elastic collisions $(e=1)$ of a particle with the boundaries. The parameters used were $r_{0}=0.4, \omega$ $=0.5, \varepsilon_{r}=\varepsilon_{R}=0.01, \varphi_{0}=\phi_{0}=\pi / 4$, and $v_{0}=0.02$. (b) Average velocity vs the number of collisions for the concentric pulsating elastic case. The saturation occurs for $\langle V\rangle \approx 0.1$.

our opinion is the most typical in the nature, and we see that the invariant tori which were above the value $-V_{\eta} \approx 0.25$, when $e=1$, now were all destroyed. The zoom in the neighborhood of $\left(\varphi,-V_{\eta}\right) \approx(2 \pi, 0.18)$, seen in Fig. 2(b), shows that the elliptic fixed point has bifurcated in a stable focus so that the attractor is visible. We emphasize that as the dissipation is weak enough, there is stickiness (see more about stickiness in [19-22]) along the phase space and that many initial conditions (IC) spend a long time orbiting the attracting fixed point before being captured. We can see in Fig. 2(c) that the average velocity has saturated around $\langle V\rangle \approx 0.17$ ( $\approx 1 / 2 \pi$ ), thus leading to a gain around $\sim 70 \%$ for the average velocity of the particle in comparison with the nondissipative regime.

The calculation of the average velocities was developed in fact through two averages. First we chose a line with $k$ $=10^{3} \mathrm{IC}$, in the bottom of the phase space, for $-V_{\eta}=0.02$ and $\varphi=[0,4 \pi]$, so that for the $j$ th IC we iterate the maps and we 

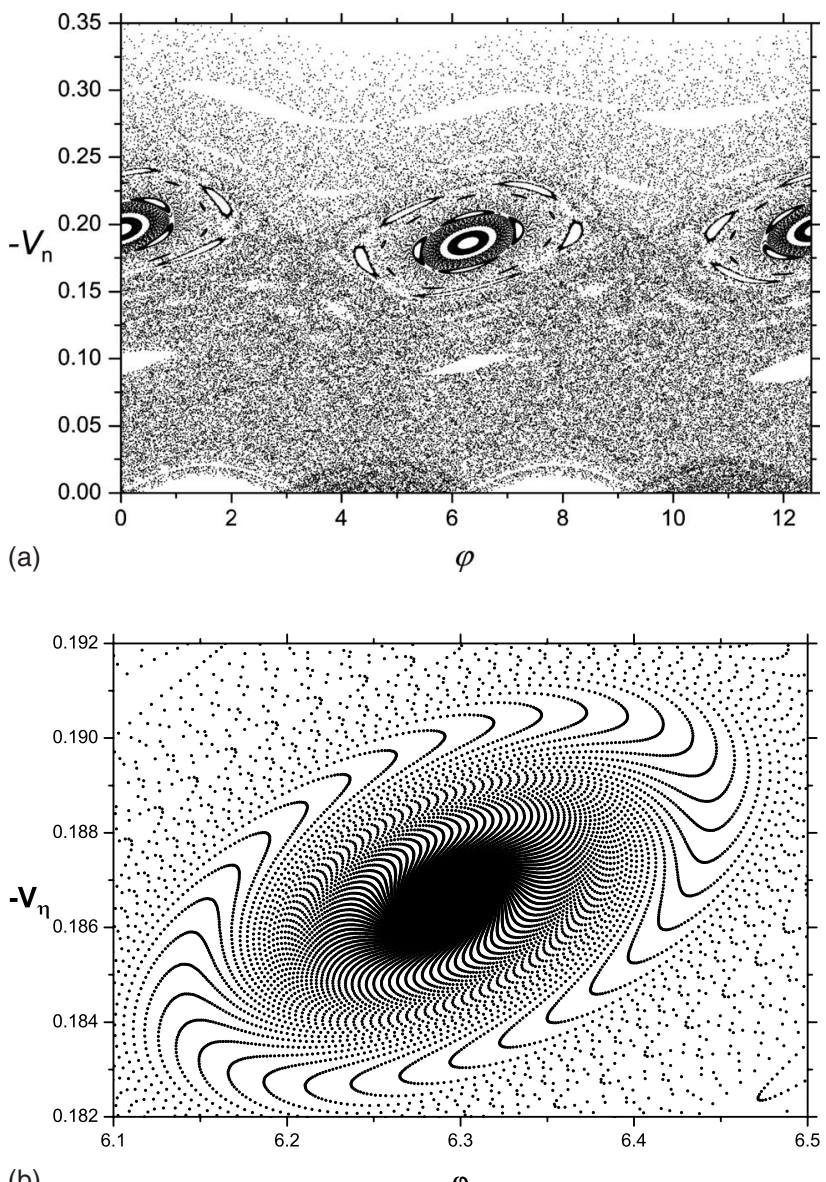

(b)

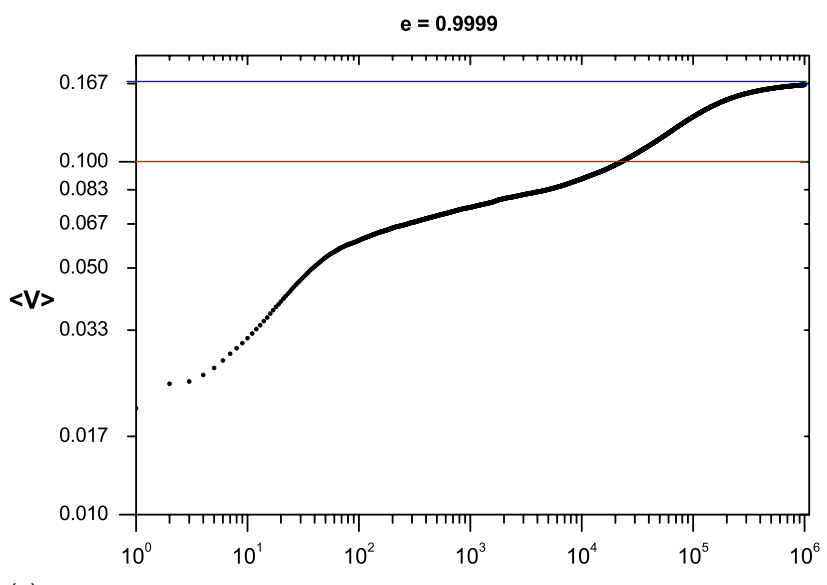

(c)

FIG. 2. (Color online) (a) Phase space for the concentric pulsating annular billiard representing inelastic collisions $(e=0.9999)$ of a particle with the boundaries. The parameters used were the same as Fig. 1(a). (b) Zoom in the neighborhood of the attractive fixed point whose velocity is $\approx 0.19$. (c) Average velocity vs the collision number for the pulsating inelastic case. The saturation occurs for $\langle V\rangle$ $\approx 0.16$, which is a higher value than the elastic pulsating case.

make an average along the orbit in terms of the number of iterations: $\left\langle V_{j}\right\rangle=(1 / n) \sum_{i=1}^{n} V_{i, j}$ up $n=10^{6}$ iterations. After repeating this first average for all IC, we construct an ensemble of $\left\langle V_{j}\right\rangle$, and from there we perform the average along the
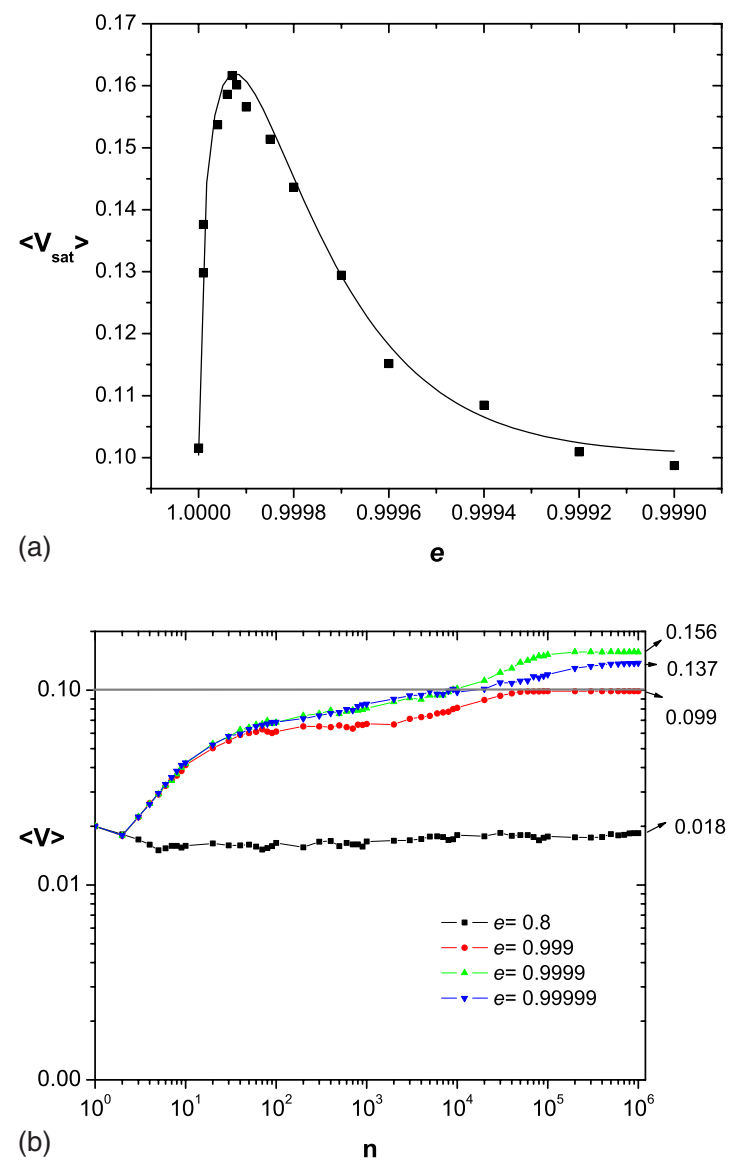

FIG. 3. (Color online) (a) Average velocity vs the collision number for four values of the restitution coefficient. (b) Average saturation velocity of the particle as function of the restitution coefficient exhibiting a range of $e$ where the particle can gain energy. A nonlinear curve fits the data very well.

ensemble: $\langle V\rangle=(1 / k) \sum_{j=1}^{k}\left\langle V_{j}\right\rangle$. This average velocity is plotted in Figs. 1(b) and 2(c). As this attractor occurs for a velocity $[\approx 0.19$ in Fig. 2(a)] above the value of the average velocity for the nondissipative case $[\langle V\rangle \approx 0.1$ in Fig. 1(b)], the system experiences a gain on its average energy. So this procedure constitutes a mechanism that may provide an increasing on the average energy of any weakly dissipative dynamical system, which should satisfy the dynamical conditions described here.

We also point out that this scenario may be destructed if the dissipation becomes stronger. In Fig. 3(a) we present four plots for the average velocity of the particle as function of the collision number, which show that it is possible to have energy gain in a certain interval of the restitution coefficient. For $e=0.999$ the average velocity saturates near the value of the conservative case, $\langle V\rangle \sim 0.1$, for $e=0.9999$ and $e$ $=0.99999,\langle V\rangle$ attains higher values showing gain of energy and for $e=0.8$ the dissipation is very strong and there is loss of energy. In order to verify the dependence of the average saturation velocity of the particle, $\left\langle V_{\text {sat }}\right\rangle$, with respect to the restitution coefficient $e$, we have performed the averages cited above for 15 values of $e$, around the value of $e$ for which we have observed the energy gain. In Fig. 3(a) we 
show the behavior of $\left\langle V_{\text {sat }}\right\rangle$ in terms of $e$, where the squares correspond to the numerical data that we have computed and the continuous curve corresponds to a nonlinear fitting of the type

$$
\left\langle V_{\text {sat }}\right\rangle_{e}=\left\langle V_{\text {sat }}\right\rangle_{e 0}+a\left[1-\exp \left(\frac{e-e_{0}}{b}\right)\right]^{P} \exp \left(\frac{e-e_{0}}{c}\right),
$$

where the parameters have assumed the values $a=1.567, b$ $=2.634 \times 10^{-2}, c=1.6 \times 10^{-4}, P=0.472, e_{0}=1$ (conservative case), and $0.999 \leqslant e \leqslant 1$. We observe that the system presents a growth in its average energy until a pick around $e$ $\sim 0.99993$, which corresponds to $\langle V\rangle \sim 0.162$, and from there the system still gains energy but in a decreasing fashion up $e \sim 0.999$. For smaller values of the restitution coefficient, or stronger dissipation, the behavior of the system does not obey this fitting curve anymore and there is not any other interval of $e$ which presents energy gain.

We would like to emphasize that, based on our investigations, this mechanism of energy gain depends strongly on the phase space architecture. We only have observed this energy gain when (i) the resonance structures, which will generate the attractors, have velocities higher than the saturation velocity of the conservative case, (ii) the resonance structures are immerse in the chaotic sea, and (iii) the phase space does not present any spanning curve between this chaotic sea and the velocities chosen as initial conditions. Based on these observations we evaluate that our results are sufficiently generic to be observed in many other dynamical systems.

As concluding remarks, we point out that our choice of a weakly dissipative system followed the ideas presented in [23] where, among others, it is posed that in practice the systems are more often neither strongly dissipative nor conservative. In this intermediary regime, the inelasticity on the annular billiard boundaries may be considered as a small perturbation which can be used to control chaos or to stabilize regular dynamical behaviors [24], besides providing a gain in the average energy for the particle as reported in this paper. In that sense, even though our approach had been developed in a particular model, it constitutes a general mechanism that can be used in different fields of science.

\section{ACKNOWLEDGMENTS}

The authors acknowledge fruitful discussions with E. D. Leonel and also thank the Brazilian scientific agency FAPESP for partial financial support through the process 06/ 51653-0 and 06/56477-6.
[1] G. Katz, M. A. Ratner, and R. Kosloff, Phys. Rev. Lett. 98, 203006 (2007).

[2] S. Bohn and M. O. Magnasco, Phys. Rev. Lett. 98, 088702 (2007).

[3] M. Durand, Phys. Rev. Lett. 98, 088701 (2007).

[4] N. Vermeulen, C. Debaes, P. Muys, and H. Thienpont, Phys. Rev. Lett. 99, 093903 (2007).

[5] P. Perlekar, D. Mitra, and R. Pandit, Phys. Rev. Lett. 97, 264501 (2006).

[6] T. Cubaud and T. G. Mason, Phys. Rev. Lett. 96, 114501 (2006).

[7] J. E. Avron, B. Gutkin, and D. H. Oaknin, Phys. Rev. Lett. 96, 130602 (2006).

[8] A. Zazunov, M. Creux, E. Paladino, A. Crépieux, and T. Martin, Phys. Rev. Lett. 99, 066601 (2007).

[9] K. Yu. Bliokh, Yu. P. Bliokh, V. Freilikher, A. Z. Genack, B. Hu, and P. Sebbah, Phys. Rev. Lett. 97, 243904 (2006).

[10] L. Baiotti and L. Rezzolla, Phys. Rev. Lett. 97, 141101 (2006).

[11] H. C. Fu, A. Seidel, J. Clarke, and Dung-Hai Lee, Phys. Rev. Lett. 96, 157005 (2006).

[12] A. Kis, K. Jensen, S. Aloni, W. Mickelson, and A. Zettl, Phys.
Rev. Lett. 97, 025501 (2006).

[13] R. Tenny, Lev S. Tsimring, L. Larson, and H. D. I. Abarbanel, Phys. Rev. Lett. 90, 047903 (2003).

[14] E. Ott, Chaos in Dynamical Systems (Cambridge University Press, Cambridge, UK, 1993).

[15] N. Saito, H. Hirooka, J. Ford, F. Vivaldi, and G. H. Walker, Physica D 5, 273 (1982).

[16] O. Bohigas, D. Boose', R. Egydio de Carvalho, and V. Marvulle, Nucl. Phys. A 560, 197 (1993).

[17] R. Egydio de Carvalho, F. Caetano de Souza, and Edson D. Leonel, J. Phys. A 39, 3561 (2006).

[18] R. E. de Carvalho, F. C. Souza, and E. D. Leonel, Phys. Rev. E 73, 066229 (2006).

[19] J. D. Meiss and E. Ott, Phys. Rev. Lett. 55, 2741 (1985).

[20] J. D. Hanson, J. R. Cary, and J. D. Meiss, J. Stat. Phys. 39, 327 (1985).

[21] C. F. F. Karney, Physica D 8, 360 (1983).

[22] G. M. Zaslavsky, Phys. Rep. 371, 461 (2002).

[23] U. Feudel and C. Grebogi, Phys. Rev. Lett. 91, 134102 (2003).

[24] T. Shinbrot, C. Grebogi, J. A. Yorke, and E. Ott, Nature (London) 363, 411 (1993). 